\title{
Experimental Models of Brain Injury
}

\author{
Hadi Aligholi ${ }^{1,2 *}$, Maryam Safahani ${ }^{1,3}$ \\ ${ }^{1}$ Shefa Neuroscience Research Center, Khatam Alanbia Hospital, Tehran, Iran
}

${ }^{2}$ Department of Neuroscience, School of Advanced Medical Sciences and Technologies, Shiraz University of Medical Sciences, Shiraz, Iran

${ }^{3}$ Department of Nutrition, School of Nutritional Sciences and Dietetics, Tehran University of Medical Sciences, Tehran, Iran

\section{A BSTRACT}

Introduction: Brain injury is one of the main concerns in societies and a leading cause of death and disability worldwide. Following a brain injury, primary and secondary injuries occur and cause a complicated pathological process. Experimental brain injury models can be divided into focal brain injury models, diffused brain injury models, models used in tissue engineering strategies, and in vitro models of brain injury. Conclusion: By choosing a proper experimental model, we can address the pathology of brain injury and assess the potential treatments.

* Corresponding Author: Hadi Aligholi

E-mail: hadialigholi@yahoo.com 


\title{
مدل هاى تجربى آسيب مغزى
}

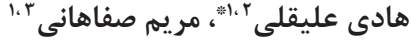 \\ 'مركز تحقيقات علوم اعصاب شفا، بيمارستان خاتم الانبياء، تهران، ايران

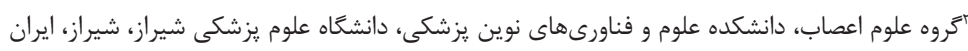

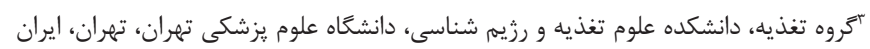

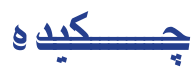

مقدمه: آسيب مغزى يكى از نكرانى هاى جدى جوامع و علت عمدهُ مركى و ناتوانى در سر تاسر جهان است.

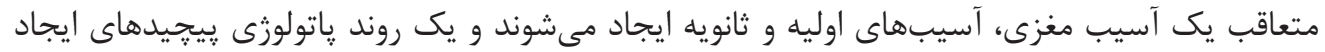

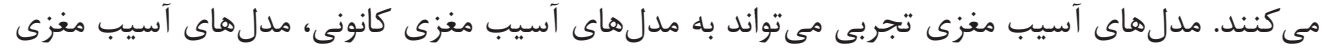

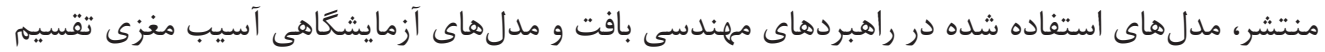

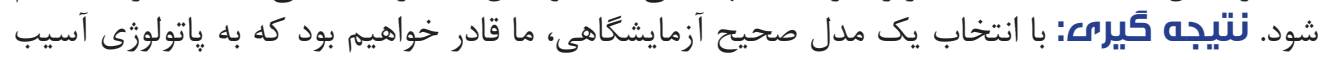

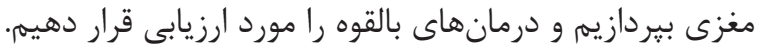


نفوذ يك جسم نافذ مثل جاقو يا گَلوله و يا مواجهه با امواج

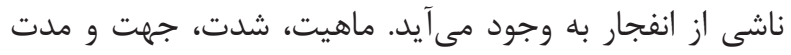

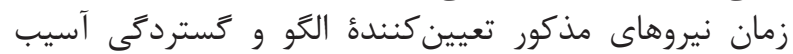

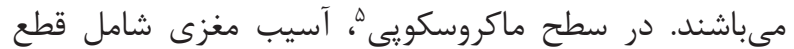

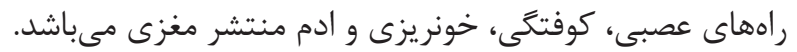
در سطح سلولى، صدمه به غشاء سلونه

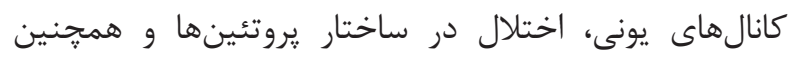

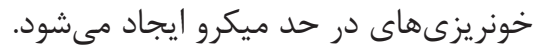

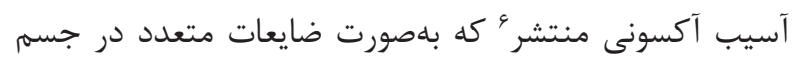

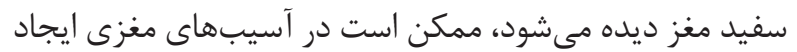

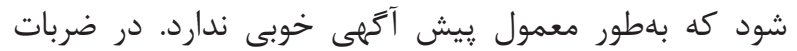

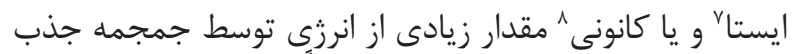

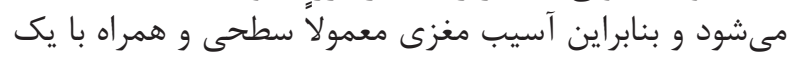

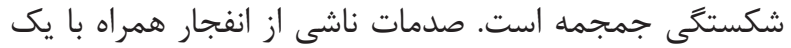

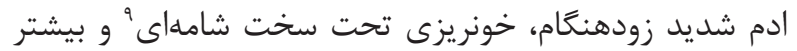

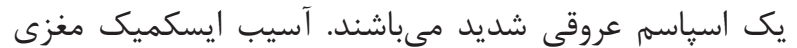

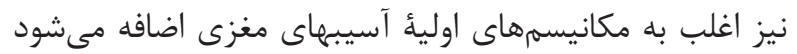

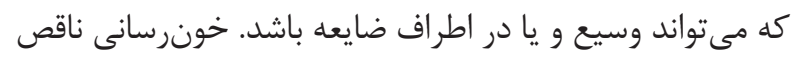

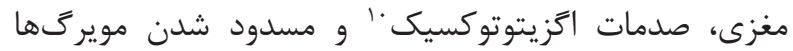

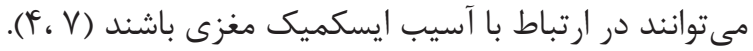
r-r هر يك از انواع صدمه به سر ممكن است مكانيسمهاى

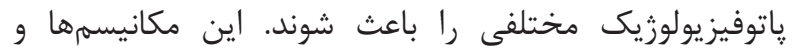

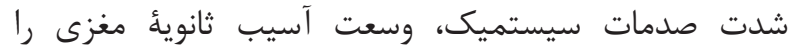

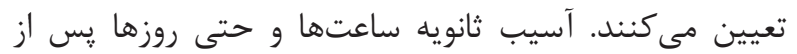

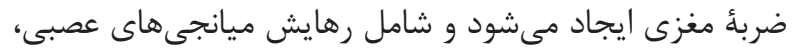

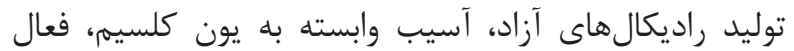

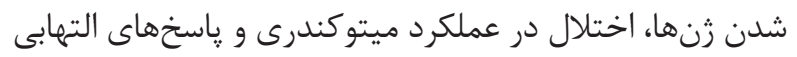

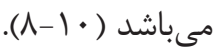

كلوتامات و ديگر ميانجى هاى عصبى تحريكى موجب نشت بيش انش

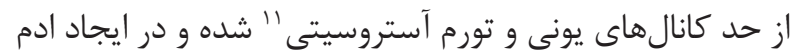

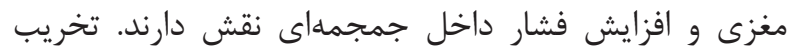

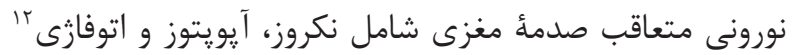

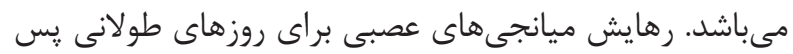

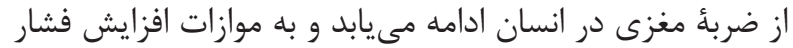

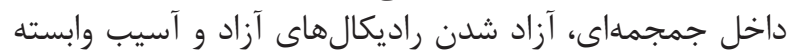

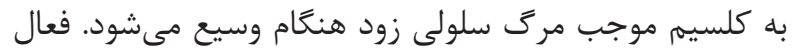

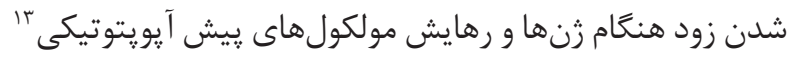

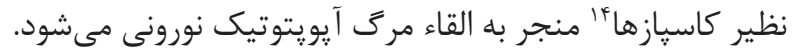

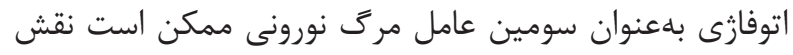

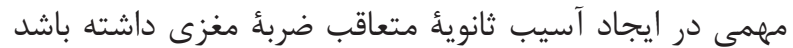

${ }^{1}$ Epigenetic

${ }^{2}$ Contusion

${ }^{3}$ Closed head injury

${ }^{4}$ Penetrating brain injury

${ }^{5}$ Macroscopic

${ }^{6}$ Diffuse axonal injury

${ }^{7}$ Static
مقدمه - مق

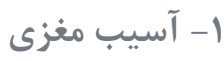

آسيب مغزى يكى از مشكلات جدى اجتماعى -اقتصادى جوامع

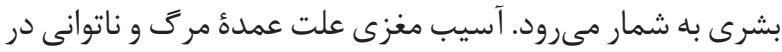

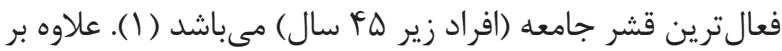

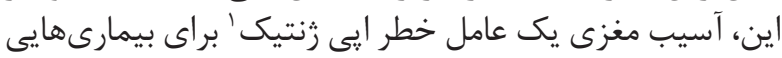

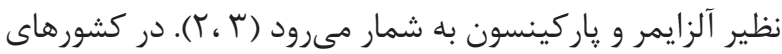

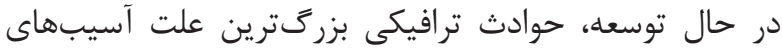

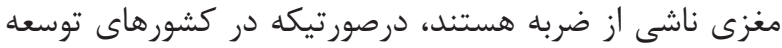

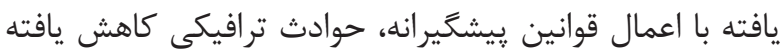

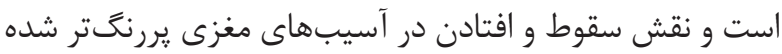

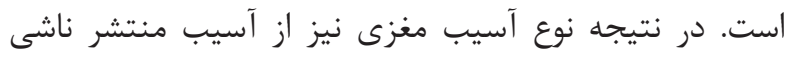

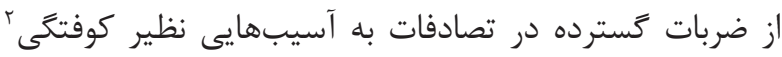

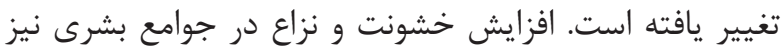

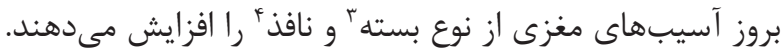

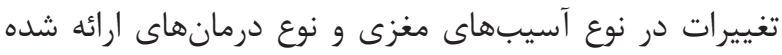

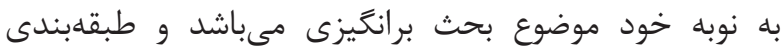

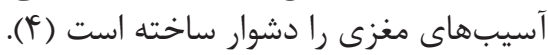

$$
\text { r - إيبدميولوزى آسيبهاى مغزى }
$$

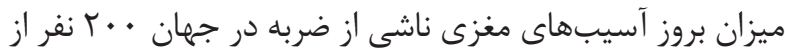

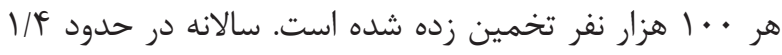

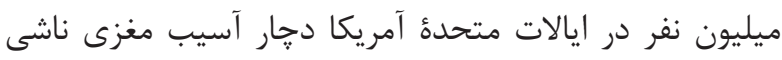

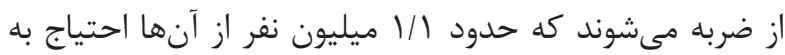

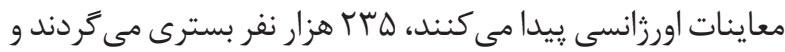

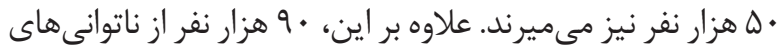

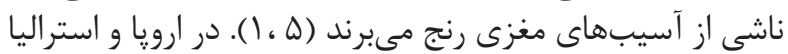

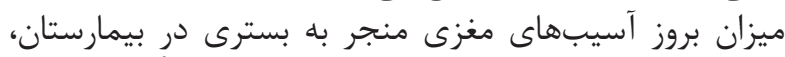

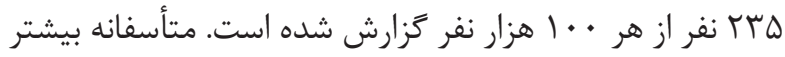

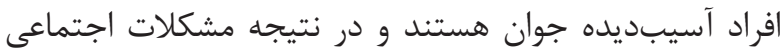

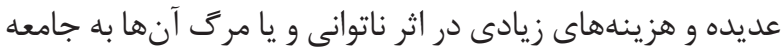

تحميل مىشود (Y). (Y).

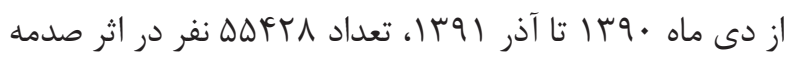

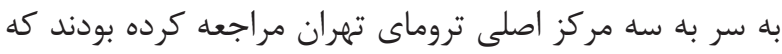

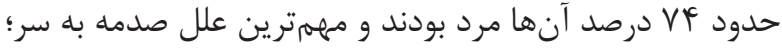

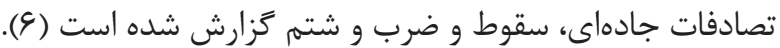

$$
\text { r- ب- انواع آسيب مغزى }
$$

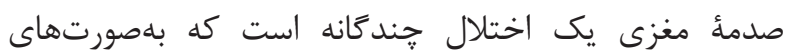
خوناگونى ظاهر مى شود. آسيب مغزى در در اثر اعمال يكى نيروى

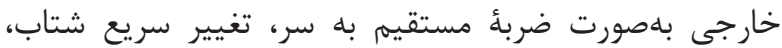

\footnotetext{
${ }^{8}$ Focal

${ }^{9}$ Subarachnoid haemorrhage

${ }^{10}$ Exitotoxic

${ }^{11}$ Astrocytic swelling

${ }^{12}$ Autophagy

${ }^{13}$ Preapoptotic

${ }^{14}$ Caspases
} 


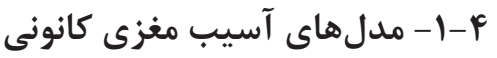

مل ملهاى آسيب مغزى كانونى شامل Weight Drop Model (IV) Controlled Cortical Impact (CCI) Model (19)

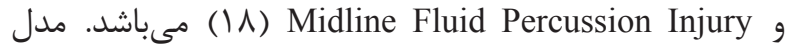
Weight Drop

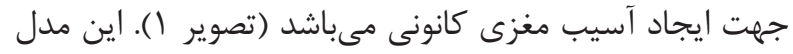

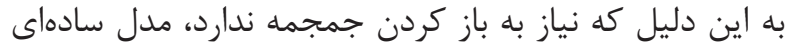

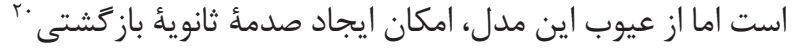

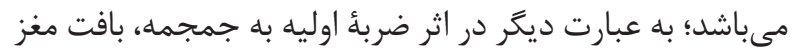

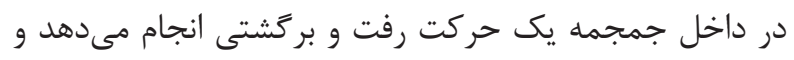

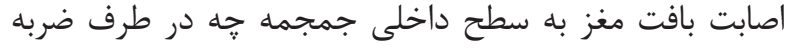

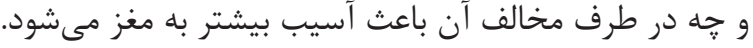
صدمأ ثانويأ بازگشتى نوعى ديخر از صدمأ كانونى مدل CCI است

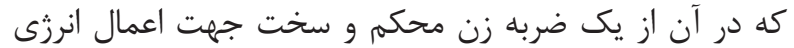

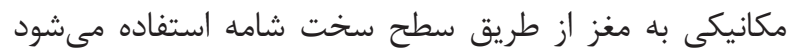

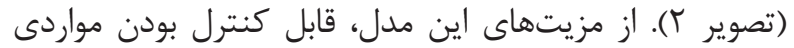

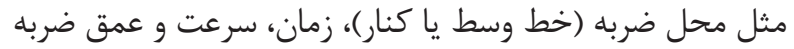

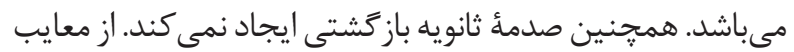

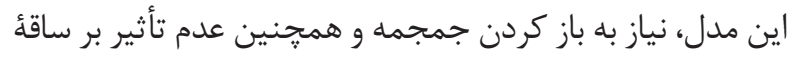

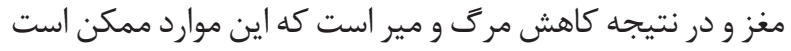
متفاوت با وضعيت طبيعى در انسان باشد.

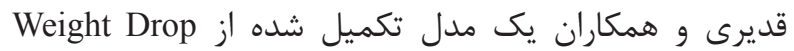

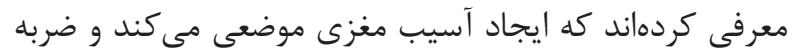

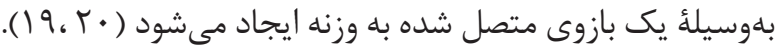

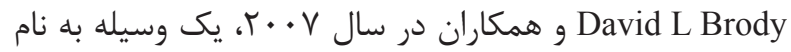
Electromagnetic Controlled Cortical Impact كه بلوسيلة نيروى الكترومغناطيس يك ضر بأه مشخص و كنترل

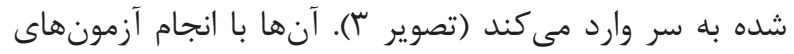

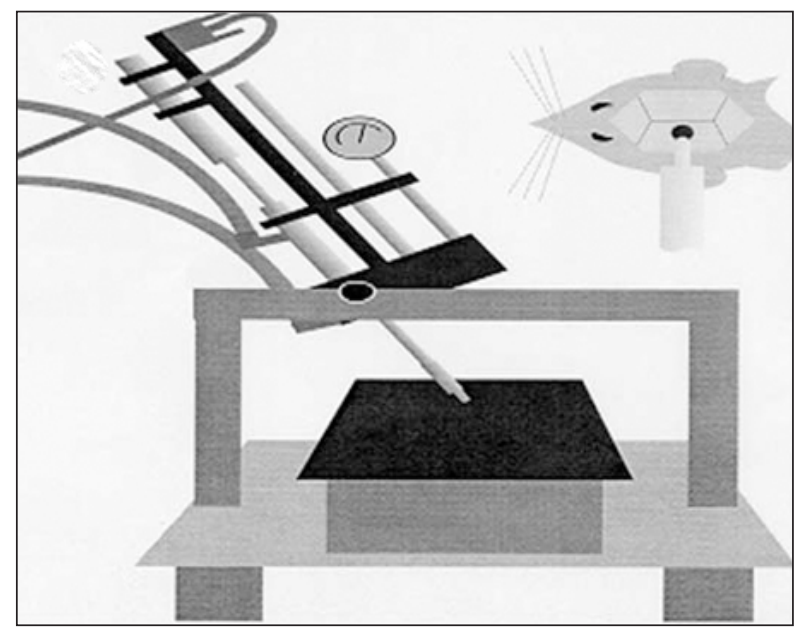

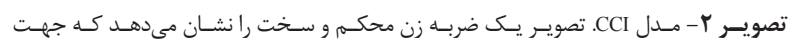

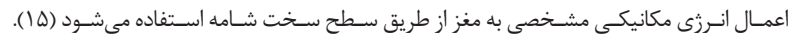

\footnotetext{
${ }^{15}$ Regeneration

${ }^{16}$ Adenosine thriphosphate

${ }^{17}$ Mitochondrial transition pore
}

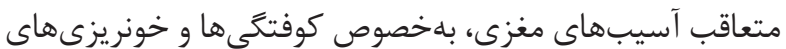

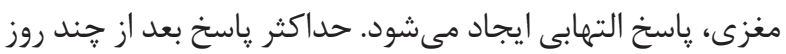

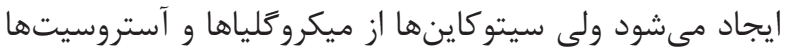

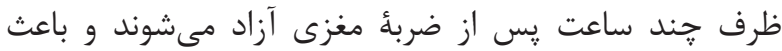

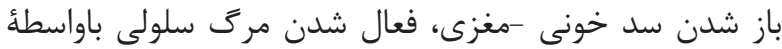

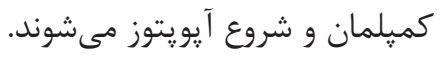

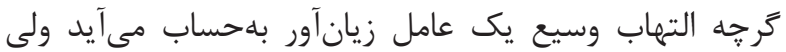

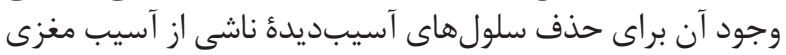

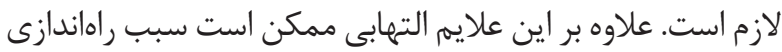

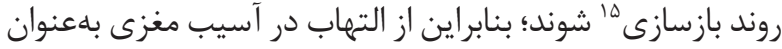

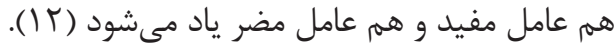

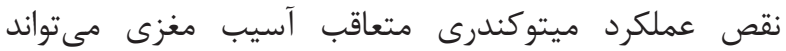

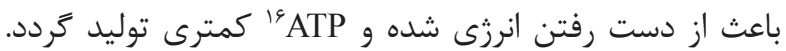

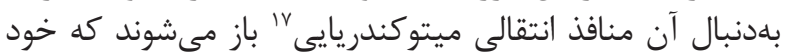

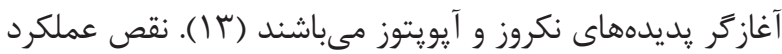

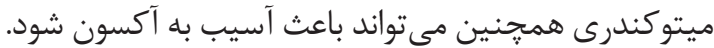

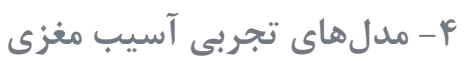

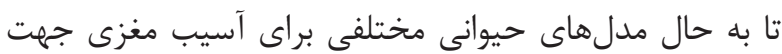

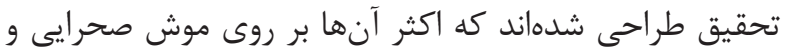

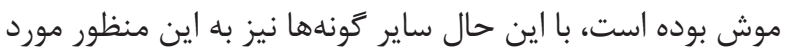

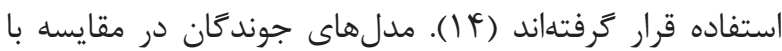

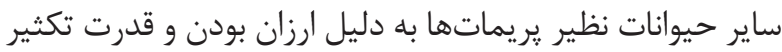

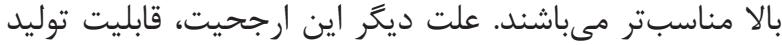

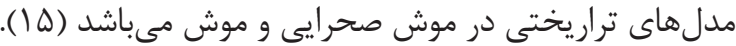

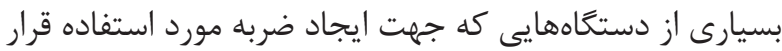

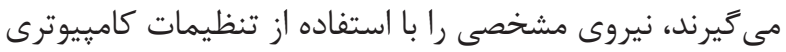

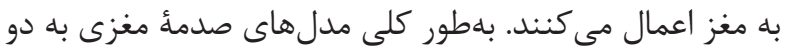

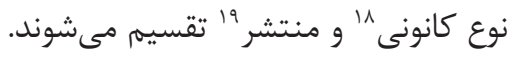

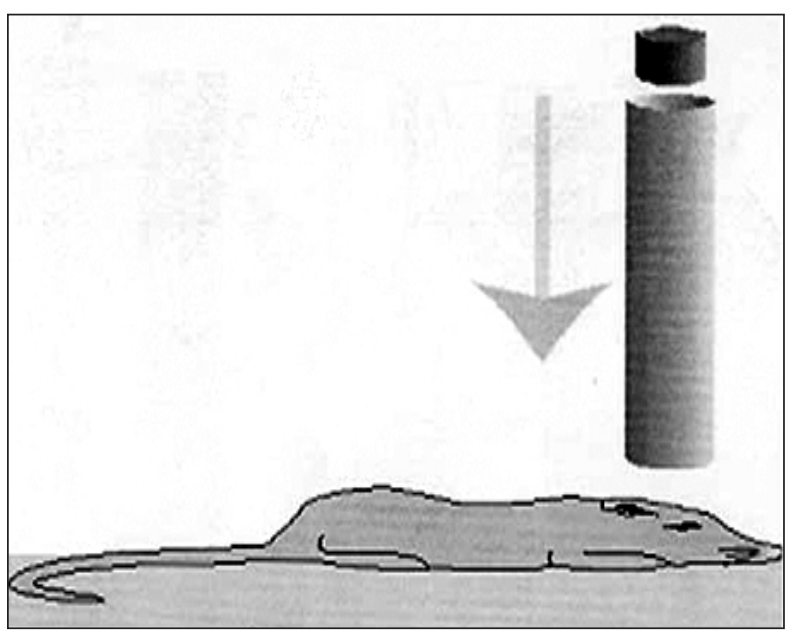

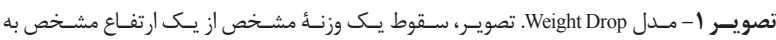

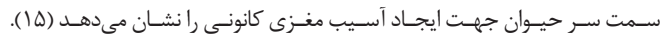
${ }^{18}$ Focal injury
${ }^{19}$ Diffuse injury
${ }^{20}$ Secondary rebound injury 


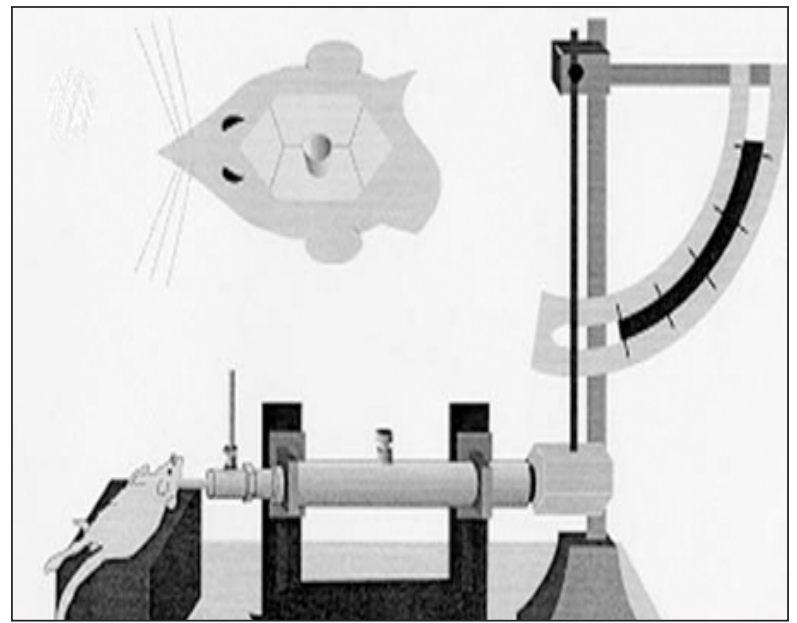

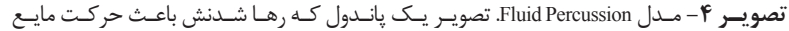

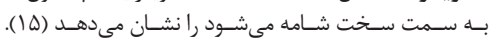

اين مدل موجب كوفتگىى مغزى، هماتوم تحت سخت شامه و

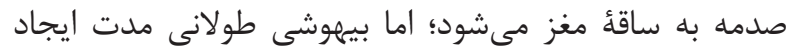

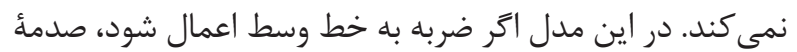

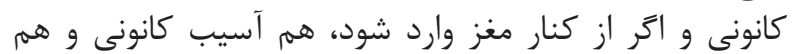

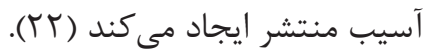

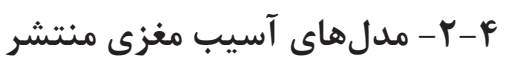
مدلهاى آسيب مغزى منتشر جهت شبيدسازى صدمات بالينى مانى

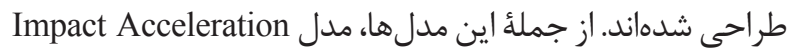

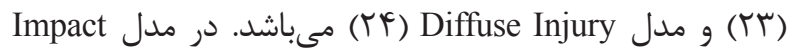
Acceleration

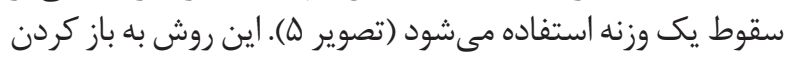

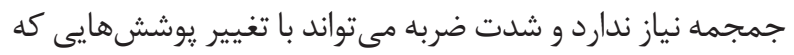

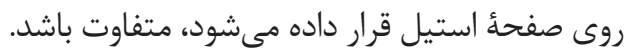

اين مدل موجب صدمةٔ اكسونى و كما مىشود. در مدل Diffuse

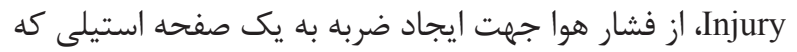

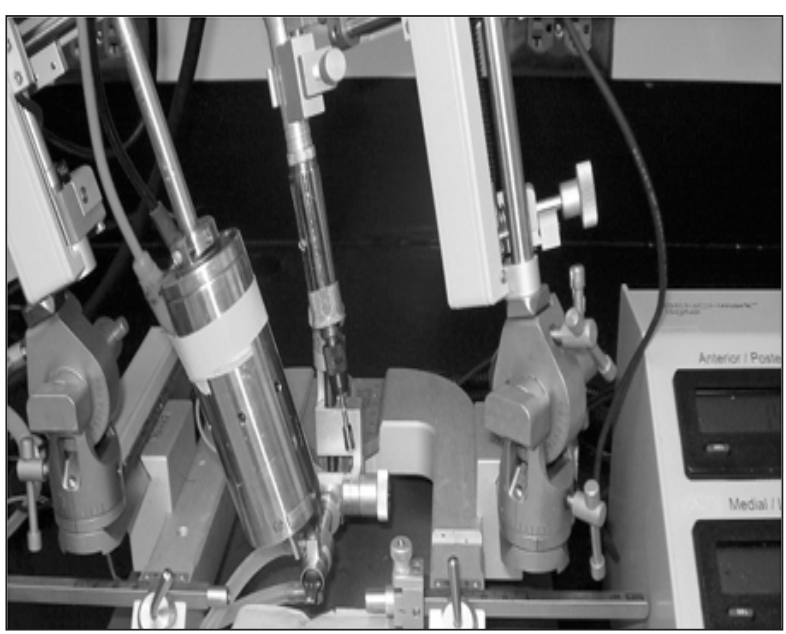

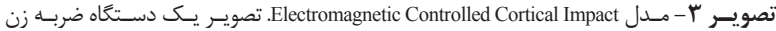

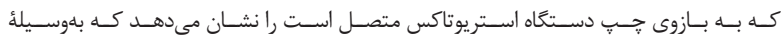

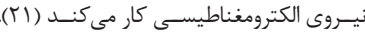

رفتارى و يروهشهاى بافتشناسى ثابت كردهاند كه دستخاه

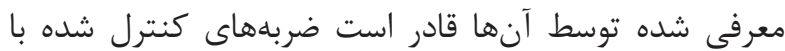
شدتها و آسيبهاى متفاوت توليد كند (Y) (Y). در اين مطالعه، عمق ضربه بين ا تا ץ ميلىمتر در نظر گرفته

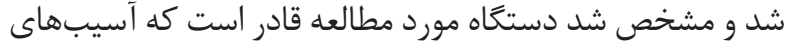

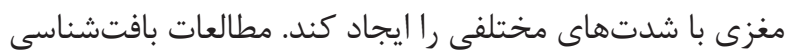

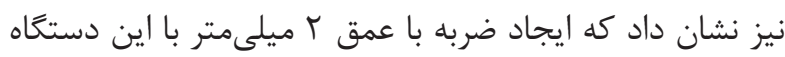

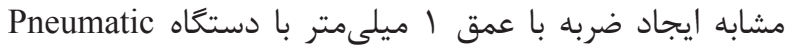

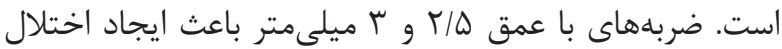

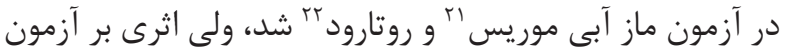
ترس شرطى شده نداشت.

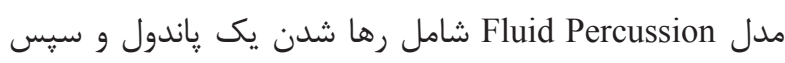

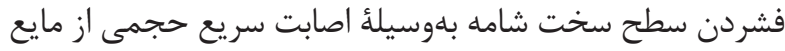

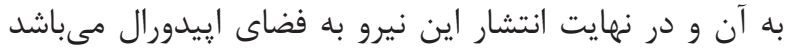

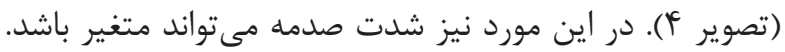

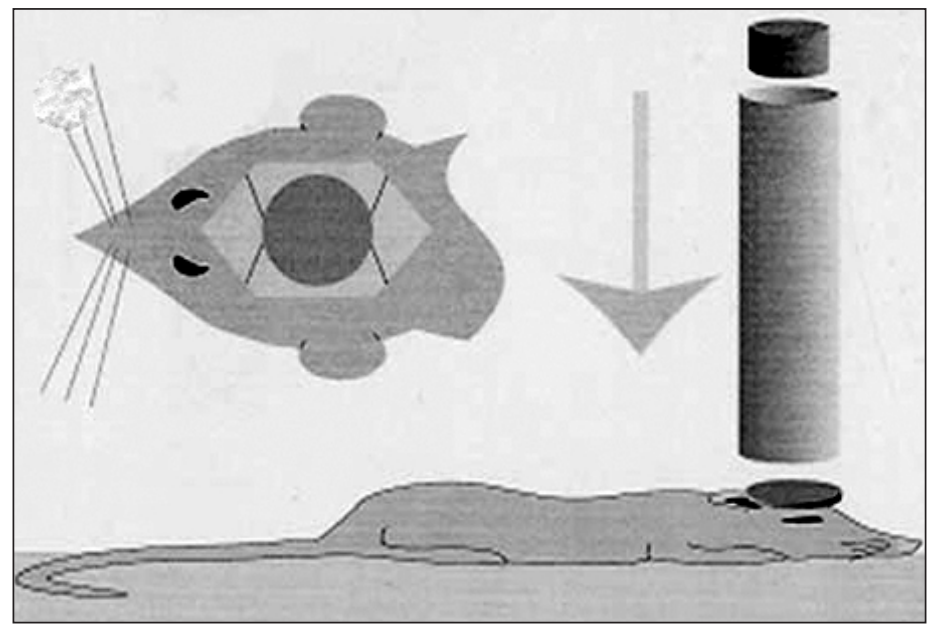

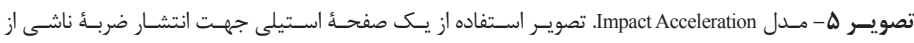

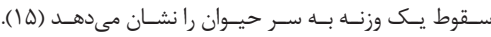

\footnotetext{
${ }^{21}$ Morris Water Maze
}

${ }^{22}$ Rotarod test 


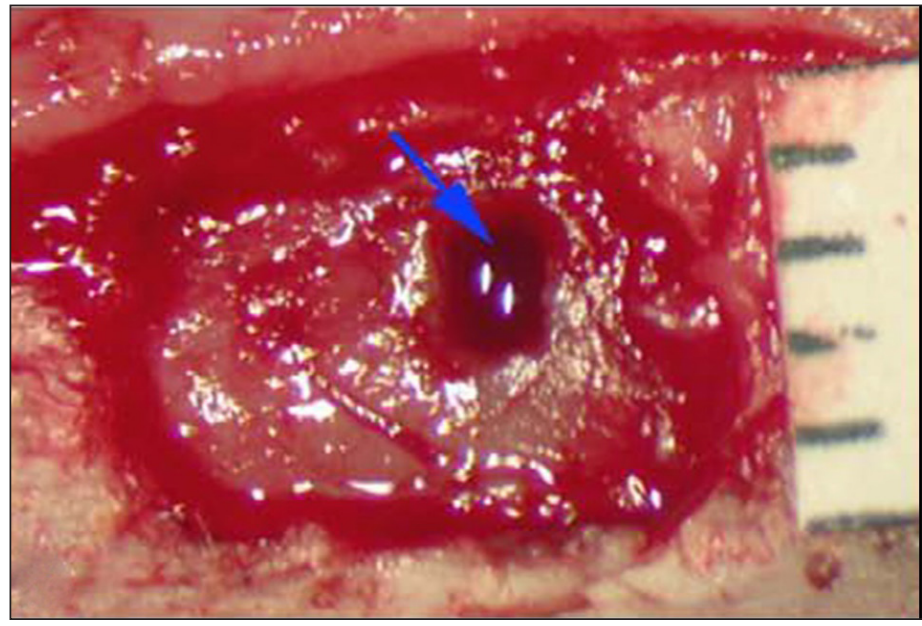

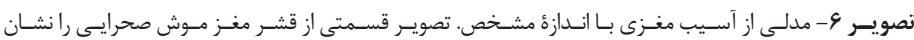

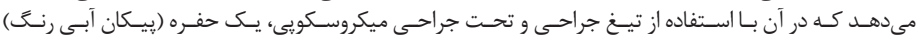

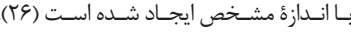

يك لوله فلزى استوانهاى است كه يك طرف آن مسدود شده

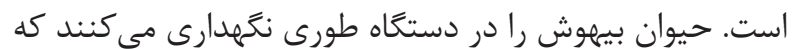

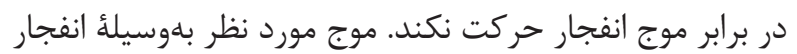

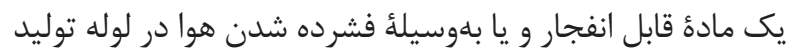

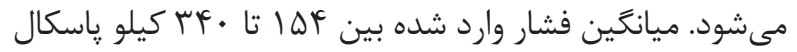

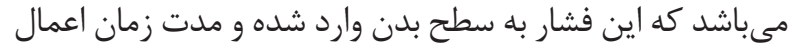

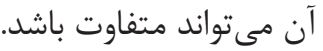

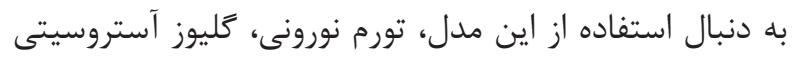

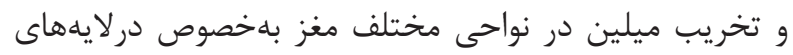

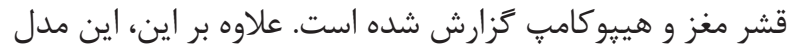

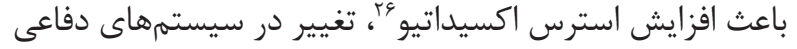
آنتىاكسيدانى، افزايش متابوليسم اكسيد اكسيد نيتريك و و سرانجام

آشفتگى هاى شناختى مىشود (TV، YN).

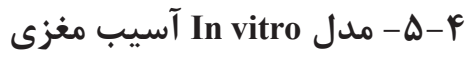

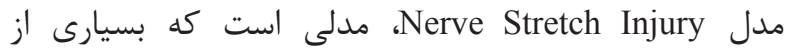

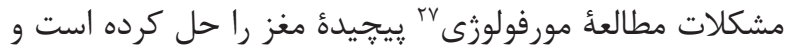

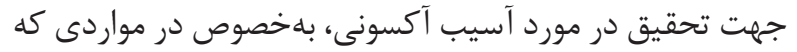

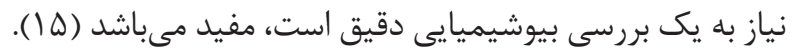

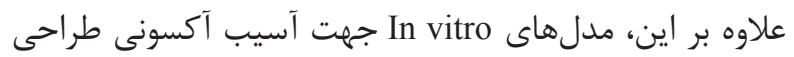

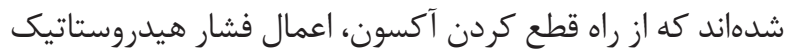

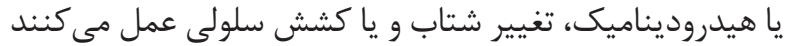

بسيارى از اين مدلهاى In vitro باعث تغيير ساختمان

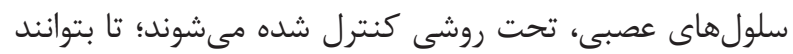

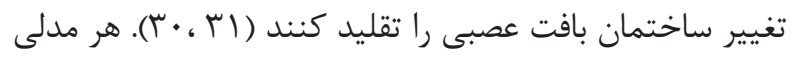

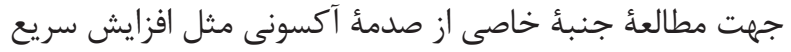

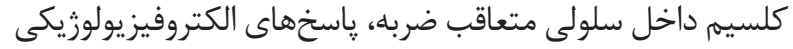

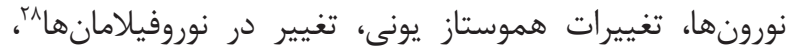

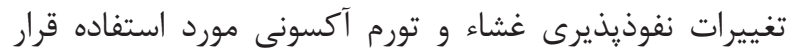

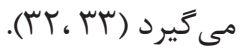

\footnotetext{
${ }^{23}$ Microscopic

${ }^{24}$ Blast traumatic brain injury

${ }^{25}$ Dynamic
}

روى مغز گذاشته شده، استفاده مىشود. سر حيوان بلوسيلة

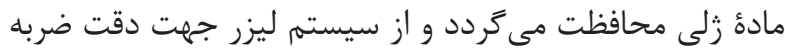

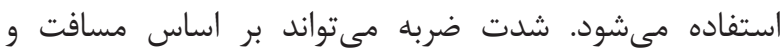

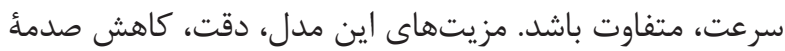

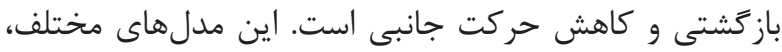

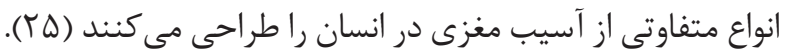

\section{F-F-F - مدلى جهت بر رسى محصولات مهندسى بافت}

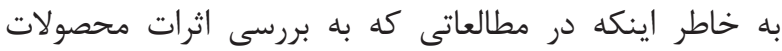

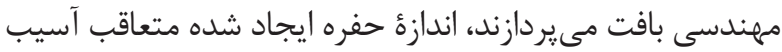

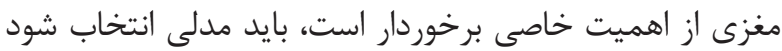

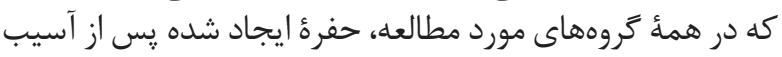

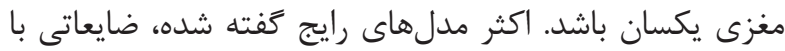

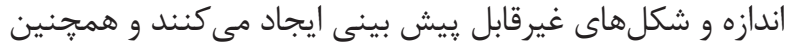

ايجاد حفره بهصورت ثانويه بعد از ضربه در بر آنها ديد ديده مى شود.

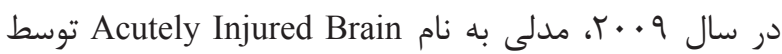
Jiasong Guo

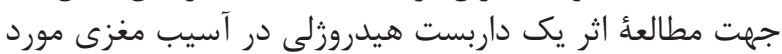

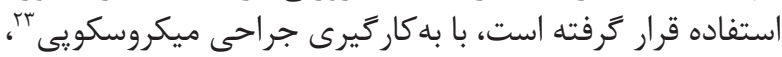

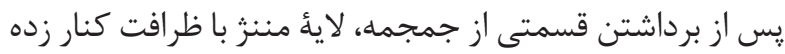

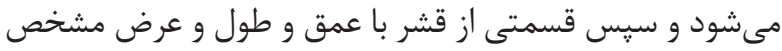

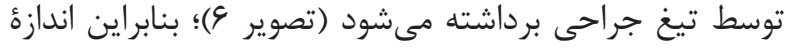

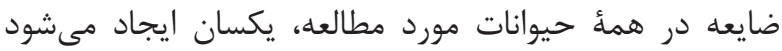

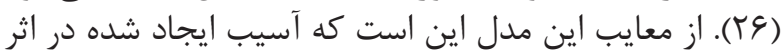

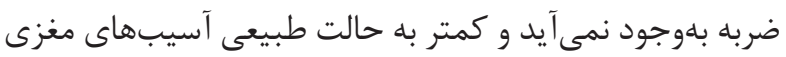

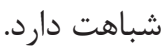

\section{- م-F-F}

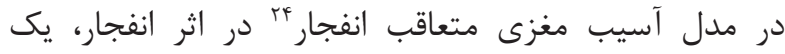

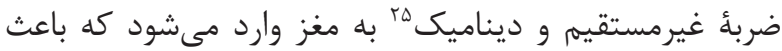

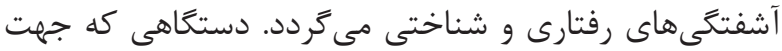

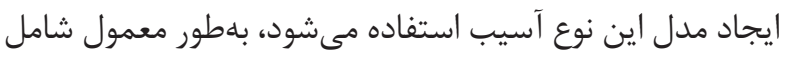

\footnotetext{
${ }^{26}$ Oxidative stress

${ }^{27}$ Morphology

${ }^{28}$ Neurofilaments
} 
مدل انتخابى به وضعيت طبيعى نزديكتر باشد، مطالعهُ ما از

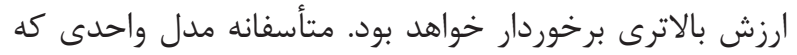

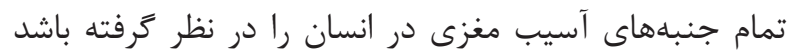

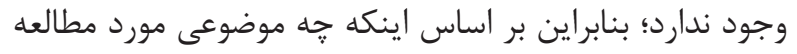

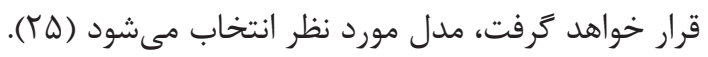

1. Langlois JA, Rutland-Brown W, Wald MM. The epidemiology and impact of traumatic brain injury: a brief overview. J Head Trauma Rehabil. 2006; 21(5): 375-8.

2. Schofield P, Logroscino G, Andrews H, Albert S, Stern Y. An association between head circumference and Alzheimer's disease in a population-based study of aging and dementia. Neurology. 1997; 49(1): 30-7.

3. Thomas LB, Gates MA, Steindler DA. Young neurons from the adult subependymal zone proliferate and migrate along an astrocyte, extracellular matrix-rich pathway. Glia. 1996; 17(1): 1-14.

4. Maas AI, Stocchetti N, Bullock R. Moderate and severe traumatic brain injury in adults. Lancet Neurol. 2008; 7(8): 728-41.

5. Aghakhani N, Azami M, Jasemi M, Khoshsima M, Eghtedar S, Rahbar N. Epidemiology of traumatic brain injury in urmia, iran. Iran Red Crescent Med J. 2013; 15(2): 173. doi: 10.5812/ircmj.2090.

6. Vafaee R, Vafaei A, Forouzanfar MM, Asadollahi S, Kashani P, Heidari K, et al. Epidemiology of traumatic brain injury in Iranian population: the results of a multicenter study.Wulfenia. 2013; 20(9): 257-63.

7. Werner C, Engelhard K. Pathophysiology of traumatic brain injury. Br J Anaesth. 2007; 99(1): 4-9.

8. Xiong $\mathrm{Y}, \mathrm{Gu} \mathrm{Q}$, Peterson P, Muizelaar J, Lee C. Mitochondrial dysfunction and calcium perturbation induced by traumatic brain injury. J Neurotrauma. 1997; 14(1): 23-34.

9. Smith SL, Andrus PK, Zhang JR, Hall ED. Direct measurement of hydroxyl radicals, lipid peroxidation, and blood-brain barrier disruption following unilateral cortical impact head injury in the rat. J Neurotrauma. 1994; 11(4): 393-404.

10. Singh IN, Sullivan PG, Deng Y, Mbye LH, Hall ED. Time course of post-traumatic mitochondrial oxidative damage and dysfunction in a mouse model of focal

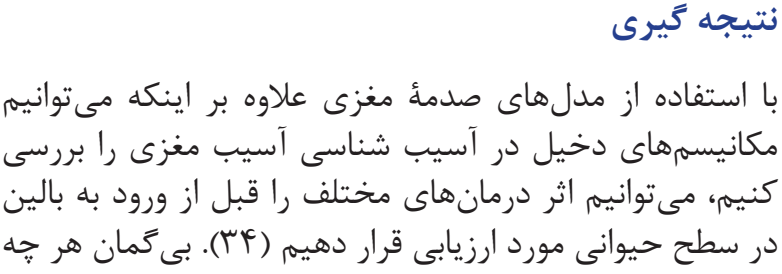

منابع

traumatic brain injury: implications for neuroprotective therapy. J Cereb Blood Flow Metab. 2006; 26(11): 1407-18.

11. Clark R, Bayir H, Chu CT, Alber SM, Kochanek PM, Watkins SC. Autophagy is increased in mice after traumatic brain injury and is detectable in human brain after trauma and critical illness. Autophagy. 2008; 4(1): 88-90.

12. Morganti-Kossmann MC, Rancan M, Stahel PF, Kossmann T. Inflammatory response in acute traumatic brain injury: a double-edged sword. Curr Opin Crit Care. 2002; 8(2): 101-5.

13. Kroemer G, Galluzzi L, Brenner C. Mitochondrial membrane permeabilization in cell death. Physiol Rev. 2007; 87(1): 99-163.

14. Webb A, Jeffery N, Olby N, Muir G. Behavioural analysis of the efficacy of treatments for injuries to the spinal cord in animals. Vet Rec. 2004; 155(8): 225-30.

15. Morales D, Marklund N, Lebold D, Thompson H, Pitkanen A, Maxwell W, et al. Experimental models of traumatic brain injury: do we really need to build a better mousetrap? Neuroscience. 2005; 136(4): 971-89.

16. Feeney DM, Boyeson MG, Linn RT, Murray HM, Dail WG. Responses to cortical injury: I. Methodology and local effects of contusions in the rat. Brain Res. 1981; 211(1): 67-77.

17. Dixon CE, Clifton G, Lighthall J, Yaghmai A, Hayes R. A controlled cortical impact model of traumatic brain injury in the rat. J Neurosci Methods. 1991; 39(3): 25362.

18. Dixon CE, Lyeth BG, Povlishock JT, Findling RL, Hamm RJ, Marmarou A, et al. A fluid percussion model of experimental brain injury in the rat. J Neurosurg. 1987; 67(1): 110-9.

19. Ghadiri T, Modarres Mousavi M, Gorji A. W4: A new animal model of traumatic brain injury in rat. Shefaye Khatam. 2015; 2(4): 47. 


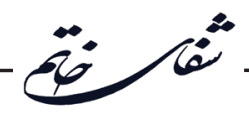

20. Ghadiri T, Sharifzadeh M, khodagholi F, Modarres Mousavi SM, Hassanzadeh G, Zarrindast MR, et al. A novel traumatic brain injury model for induction of mild brain injury in rats. J Neurosci Methods. 2014; 233: 18-27.

21. Brody DL, Mac Donald C, Kessens CC, Yuede C, Parsadanian M, Spinner M, et al. Electromagnetic controlled cortical impact device for precise, graded experimental traumatic brain injury. J Neurotrauma. 2007; 24(4): $657-73$.

22. Cortez SC, McIntosh T, Noble L. Experimental fluid percussion brain injury: vascular disruption and neuronal and glial alterations. Brain Res. 1989; 482(2): 271-82.

23. Marmarou A, Foda MAA-E, Brink Wvd, Campbell J, Kita H, Demetriadou K. A new model of diffuse brain injury in rats: part I: pathophysiology and biomechanics. J Neurosurg. 1994; 80(2): 291-300.

24. Cernak I, Vink R, Zapple DN, Cruz MI, Ahmed F, Chang $\mathrm{T}$, et al. The pathobiology of moderate diffuse traumatic brain injury as identified using a new experimental model of injury in rats. Neurobiol Dis. 2004; 17(1): 29-43.

25. Kulbatski I, Mothe AJ, Parr AM, Kim H, Kang CE, Bozkurt $\mathrm{G}$, et al. Glial precursor cell transplantation therapy for neurotrauma and multiple sclerosis. Prog Histochem Cytochem. 2008; 43(3): 123-76.

26. Guo J, Leung KKG, Su H, Yuan Q, Wang L, Chu T-H, et al. Self-assembling peptide nanofiber scaffold promotes the reconstruction of acutely injured brain. Nanomedicine. 2009; 5(3): 345-51.

27. Cernak I. Animal models of head trauma. Neuro Rx.
$2005 ; 2(3): 410-22$.

28. Koliatsos VE, Cernak I, Xu L, Song Y, Savonenko A, Crain BJ, et al. A mouse model of blast injury to brain: initial pathological, neuropathological, and behavioral characterization. J Neuropathol Exp Neurol. 2011; 70(5): 399-416.

29. Morrison B 3rd, Saatman KE, Meaney DF, Mcintosh TK. In vitro central nervous system models of mechanically induced trauma: a review. J Neurotrauma. 1998; 15(11): 911-28.

30. Dieterich DC, Böckers TM, Gundelfinger ED, Kreutz MR. Screening for differentially expressed genes in the rat inner retina and optic nerve after optic nerve crush. Neurosci Lett. 2002; 317(1): 29-32.

31. Staal J, Dickson T, Chung R, Vickers J. Cyclosporin-A treatment attenuates delayed cytoskeletal alterations and secondary axotomy following mild axonal stretch injury. Dev Neurobiol. 2007; 67(14): 1831-42.

32. Kilinc D, Gallo G, Barbee KA. Mechanically-induced membrane poration causes axonal beading and localized cytoskeletal damage. Exp Neurol. 2008; 212(2): 422-30.

33. Wolf JA, Stys PK, Lusardi T, Meaney D, Smith DH. Traumatic axonal injury induces calcium influx modulated by tetrodotoxin-sensitive sodium channels. Neurosci J. 2001; 21(6): 1923-30.

34. Nourzad Z, Khazali H, Ghadiri T, Modarres Mousavi M, Karimzadeh F, Eshaghabadi A, et al. Neuroprotective effects of concomitant use of erythropoietin and progesterone in traumatic brain injury. Shefaye Khatam. 2014; 2(2): 1-12. 